\title{
Utility of prior screening for methicillin-resistant Staphylococcus aureus in predicting resistance of $S$. aureus infections
}

Derek R. MacFadden MD, Marion Elligsen RPh, Ari Robicsek MD, Daniel R. Ricciuto MD, Nick Daneman MD MSc

\section{ABSTRACT \\ Background: Screening for methicillin- resistant Staphylococcus aureus (MRSA) is intended to reduce nosocomial spread by identifying patients colonized by MRSA. Given the widespread use of this screening, we eval- uated its potential clinical utility in predicting the resistance of clinical isolates of $S$. aureus.}

Methods: We conducted a 2-year retrospective cohort study that included patients with documented clinical infection with $S$. aureus and prior screening for MRSA. We determined test characteristics, including sensitivity and specificity, of screening for predicting the resistance of subsequent $S$. aureus isolates.

Results: Of 510 patients included in the study, 53 $(10 \%)$ had positive results from MRSA screening, and $79(15 \%)$ of infecting isolates were resistant to methicillin. Screening for MRSA predicted methicillin resistance of the infecting isolate with 99\% (95\% confidence interval [Cl] 98\%$100 \%)$ specificity and $63 \%(95 \% \mathrm{Cl} 52 \%-74 \%)$ sensitivity. When screening swabs were obtained within 48 hours before isolate collection, sensitivity increased to $91 \%(95 \% \mathrm{Cl} 71 \%-99 \%)$ and specificity was $100 \%$ (95\% Cl 97\%-100\%), yielding a negative likelihood ratio of $0.09(95 \% \mathrm{Cl}$ $0.01-0.3$ ) and a negative predictive value of $98 \%$ (95\% Cl 95\%-100\%). The time between swab and isolate collection was a significant predictor of concordance of methicillin resistance in swabs and isolates (odds ratio 6.6, 95\% Cl 1.6-28.2).

Interpretation: A positive result from MRSA screening predicted methicillin resistance in a culture-positive clinical infection with $S$. aureus. Negative results on MRSA screening were most useful for excluding methicillin resistance of a subsequent infection with $\mathrm{S}$. aureus when the screening swab was obtained within 48 hours before collection of the clinical isolate.
A ntimicrobial resistance is a global problem. The prevalence of resistant bacteria, including methicillin-resistant Staphylococcus aureus (MRSA), has reached high levels in many countries..$^{1-3}$ Methicillin resistance in $S$. aureus is associated with excess mortality, hospital stays and health care costs, ${ }^{3,4}$ possibly owing to increased virulence or less effective treatments for MRSA compared with methicillin-sensitive $S$. aureus (MSSA). ${ }^{5}$

The initial selection of appropriate empirical antibiotic treatment affects mortality, morbidity and potential health care expenditures. ${ }^{6-8}$ The optimal choice of antibiotics in $S$. aureus infections is important for 3 major reasons: $\beta$-lactam antibiotics have shown improved efficacy over vancomycin and are the ideal treatment for susceptible strains of $S$. aureus ${ }^{6} \beta$-lactam antibiotics are ineffective against MRSA, and so vancomycin or other newer agents must be used empirically when MRSA is suspected; and unnecessary use of broad-spectrum antibiotics (e.g., vancomycin) can lead to the development of further antimicrobial resistance. ${ }^{9}$ It is therefore necessary to make informed decisions regarding selection of empirical antibiotics. ${ }^{10-13}$ Consideration of a patient's previous colonization status is important, because colonization predates most hospital and community-acquired infections. ${ }^{10,14}$

Universal or targeted surveillance for MRSA has been implemented widely as a means of limiting transmission of this antibiotic-resistant pathogen. ${ }^{15,16}$ Although results of MRSA screening are not intended to guide empirical treatment, they may offer an additional benefit among patients in whom clinical infection with S. aureus develops.

Studies that examined the effects of MRSA carriage on the subsequent likelihood of infection allude to the potential diagnostic benefit of
Competing interests: None declared.

This article has been peer reviewed.

Correspondence to: Nick Daneman, nick.daneman@sunnybrook .ca

CMAJ 2013. DOI:10.1503 /cmaj.130364 
prior screening for MRSA. ${ }^{17,18}$ Colonization by MRSA at the time of hospital admission is associated with a 13-fold increased risk of subsequent MRSA infection. ${ }^{17,18}$ Moreover, studies that examined nasal carriage of $S$. aureus after documented $S$. aureus bacteremia have shown remarkable concordance between the genotypes of paired colonizing and invasive strains $(82 \%-$ 94\%). ${ }^{19,20}$ The purpose of our study was to identify the usefulness of prior screening for MRSA for predicting methicillin resistance in culturepositive S. aureus infections.

\section{Methods}

\section{Study design, setting and participants}

We conducted a retrospective cohort study of all patients admitted to Sunnybrook Health Sciences Centre between May 1, 2010, and Apr. 30, 2012, with documented clinical infection with $S$. aureus and prior screening for MRSA. Documented infection with $S$. aureus was defined as a clinical (nonsurveillance) culture that yielded S. aureus, in conjunction with physician-prescribed antistaphylococcal antibiotic therapy within 72 hours before culture collection and up to 7 days after collection. Patients 16 years of age or younger were excluded, as were patients with screening swabs obtained after the onset of infection with $S$. aureus. A patient could be included in the study only once. Only the most recently obtained swab was considered, but there was no maximum time limit between the swab being obtained and the onset of clinical infection.

Sunnybrook Health Sciences Centre is a tertiary care centre that serves about 31000 inpatients annually and offers a full range of medical, surgical and long-term care services. The Sunnybrook Research Ethics Board approved this study.

\section{Data sources}

Data were obtained from an integrated stewardship database, automatically and securely populated by microbiology, pharmacy and electronic patient care databases for all admitted patients. ${ }^{21}$

\section{Screening processes and procedures for MRSA}

According to the study centre's protocol, nares and perianal screening swabs are obtained within the first 24 hours after admission among patients who meet 1 or more of the following criteria: history of colonization by an antibiotic-resistant organism, history of contact with a patient colonized by an antibiotic-resistant organism, receipt of health care outside of Canada within the past year, admission to a health care facility within the past year, receipt of home health care or hemodialysis, residence in a shelter or inability to answer questions regarding these risk factors. Screening for MRSA is also performed on transfer to critical care units, and on any transfer or discharge in the context of an outbreak. A swab collection system by COPAN Diagnostics with charcoal transport medium was used, with culture onto chromogenic media (MRSASelect, Bio-Rad Laboratories) to screen specifically for colonies of MRSA (not MSSA). The media were incubated at $35^{\circ} \mathrm{C}$ for $22-24$ hours, and methicillin resistance was confirmed using the Vitek 2 XL system, with AST-GP67 cards (bioMérieux). A positive screening result was defined by the presence of MRSA, as detected by the above techniques, and a negative result was defined by the absence of MRSA.

\section{Processing of $\boldsymbol{S}$. aureus isolates}

Isolates were collected according to clinical context and included any of the following: urine, blood, cerebrospinal fluid, joint fluid, pleural fluid, wound or miscellaneous swab, sputum, lower respiratory sample and tissue biopsies. Sterile sites were considered to be blood, cerebrospinal fluid, peritoneal fluid, pleural fluid, synovial fluid and tissue biopsies. Culture media differed according to clinical specimen, but for all specimens, possible $S$. aureus was identified by the presence of catalase-positive, coagulasepositive colonies of gram-positive cocci, and identification and susceptibilities were confirmed by the Vitek 2 XL system, with AST-GP67 cards.

\section{Covariates}

Variables of interest collected for this study included patient characteristics (i.e., age, sex), hospital variables (i.e., ward or service at the time of clinical specimen collection), isolate variables (i.e., time of collection of the swab and clinical specimen, susceptibility results from the swab and isolate) and treatment variables (i.e., antibiotic use).

\section{Statistical analysis}

We determined test characteristics of the MRSA screening, including sensitivity, specificity, positive likelihood ratio, negative likelihood ratio, positive predictive value, negative predictive value and odds ratios (ORs), and performed stratification based on time from the swab being taken to collection of the clinical isolate. Time cut-offs were as follows: immediate (swab obtained within $48 \mathrm{~h}$ before isolate collection), recent (swab obtained between $48 \mathrm{~h}$ and $14 \mathrm{~d}$ before isolate collection) and remote (swab obtained $>14 \mathrm{~d}$ before isolate collection).

We chose these allocations to fall within 
previously demonstrated median times to colonization by $\mathrm{MRSA}^{22}$ and to permit even distribution of sample power. We considered screening for MRSA the test variable and methicillin susceptibility of the confirmed $S$. aureus clinical isolates (from a nonsurveillance location) the response variable. We defined a true positive as a positive result from MRSA screening and a clinical isolate positive for MRSA, and a false positive as a positive result from MRSA screening but a clinical isolate with MSSA. We considered a false negative to be a negative result from MRSA screening but a clinical isolate with MRSA, and a true negative to be a negative result from MRSA screening and a clinical isolate with MSSA. Precision is denoted by $95 \%$ confidence intervals (CIs) calculated using the Clopper-Pearson interval. We performed subgroup analysis on 3 identified groups: ICU versus non-ICU patients, sterile versus nonsterile sites and swabs obtained during index admission versus on prior admission.

We performed multivariable logistic regression analysis to examine predictors of concordant results between the screening swabs and clinical isolates (with respect to methicillin resistance). We defined concordance of screening and clinical isolates as either of the following: a positive result from MRSA screening and a clinical isolate with MRSA, or a negative result from MRSA screening and a clinical isolate with MSSA. We used backward subtraction to determine statistically significant model parameters, with a $p$ value threshold of $<0.05$. Subtracted covariate values (at time of removal from the model) as well as retained covariates were recorded. Goodness of fit was confirmed using the Hosmer-Lemeshow test. Statistical analysis was performed using SAS version 9.3.

\section{Results}

Of 768 patients with clinical infections with $S$. aureus diagnosed during the 2-year study period, $510(66 \%)$ had previously been screened for MRSA and were included in the study. Of the 510 screening procedures performed on these patients, $53(10 \%)$ were positive for MRSA. Of the 510 clinical isolates of $S$. aureus, 79 (15\%) were resistant to methicillin and 109 (21\%) were from a sterile site. Patient characteristics and clinical variables are shown in Table 1.

Test characteristics of MRSA screening in predicting methicillin resistance in the infecting S. aureus isolate are shown in Tables 2 and 3 . Analysis of the entire study population showed 99\% (95\% CI 98\%-100\%) specificity and 63\% (95\% CI 52\%-74\%) sensitivity.
For the subgroup of patients with less than 48 hours elapsed between the swab and isolate collection, the sensitivity of screening was $91 \%$ (95\% CI 71\%-99\%) and specificity remained high at $100 \%$ (95\% CI 97\%-100\%). Sensitivity declined as the time from swab collection to isolate collection increased; however, the test characteristics remained similar from recent ( $48 \mathrm{~h}-14 \mathrm{~d}$ ) to remote (> $14 \mathrm{~d}$ ) groups. In a further subgroup analysis, there was a significant decline in sensitivity (44\% [95\% CI 31\%-62\%]) for swabs obtained on a separate admission from the admission with the clinical $S$. aureus infection, but specificity was preserved in this subgroup at $97 \%$ (95\% CI 89\%-100\%).

Table 1: Demographic and clinical variables among 510 patients with clinical isolates that tested positive for Staphylococcus aureus and prior screening for methicillin-resistant $S$. aureus

Variable No. (\%) of patients*

Age, yr, mean \pm SD $63 \pm 28$

Sex

Male

$332(65)$

Female

178 (35)

Screening result for MRSA

Positive

$53(10)$

Negative

$457(90)$

Methicillin resistance of $S$. aureus clinical isolates

Resistant

79 (15)

Susceptible

431 (85)

Admission category

Medical

$240(47)$

Surgical

270 (53)

Admitted to intensive care unit†

207 (41)

Swab and isolate collection done on same admission 429 (84)

Clinical cultures obtained from specific siteł

Sterile

109 (21)

Blood

Fluid

$21 \quad(4)$

Tissue biopsy

26 (5)

Nonsterile

401 (79)

Lower respiratory 156 (31)

Urine $\quad 45 \quad$ (9)

Swab/miscellaneous

$200(39)$

Time between swab and isolate collection

Immediate ( $<48 \mathrm{~h})$

149 (29)

Recent (48 $h$ to $14 d$ )

233 (46)

Remote (> $14 \mathrm{~d}$ )

128 (25)

Time from admission to isolate collection, $d$, mean (IQR) $15(10-20)$

Time from swab to isolate collection, $d$, mean \pm SD $18 \pm 14$

Antibiotic treatment between swab and isolate collection

Note: $\mathrm{IQR}$ = interquartile range, MRSA = methicillin-resistant Staphylococcus aureus,

$\mathrm{SD}=$ standard deviation.

*Unless stated otherwise.

tAt the time of isolate collection

‡Sterile sites include sterile space fluids (e.g., pleural, cerebrospinal) and tissue biopsies. 
In the multivariate logistic regression analysis, no significant collinearity was detected among putative predictors (Table 4). The only statistically significant covariate associated with concordant screening and isolate results was a time of less than 48 hours between swab and isolate collection (OR 6.6 [95\% CI 1.6-28.2]). There was a trend toward increased concordance for swabs and isolates collected during the same admission (OR 2.1 [95\% CI 0.9-4.6]), but only the covariate of time between swab and isolate collection of less than 48 hours was retained in the final model (Table 4).

For the subgroup of patients with a swab obtained within 48 hours of isolate collection, $92 \%$ had screening results available before

Table 2: Test characteristics of screening to predict methicillin resistance in clinical isolates of Staphylococcus aureus, stratified by time from screening swab to isolate collection

\begin{tabular}{|c|c|c|c|c|}
\hline Characteristic & Overall & Immediate* & Recent $†$ & Remoteł \\
\hline True positive, no. & 50 & 20 & 17 & 13 \\
\hline True negative, no. & 428 & 127 & 203 & 98 \\
\hline False positive, no. & 3 & 0 & 1 & 2 \\
\hline False negative, no. & 29 & 2 & 12 & 15 \\
\hline Sensitivity, \% (95\% Cl) & $63(52-74)$ & 91 (71-99) & $59(39-77)$ & $46(28-66)$ \\
\hline Specificity, \% (95\% Cl) & $99(98-100)$ & $100(97-100)$ & $100(97-100)$ & $98(93-100)$ \\
\hline Positive likelihood ratio $(95 \% \mathrm{Cl})$ & $91(29-284)$ & $-\S$ & $120(17-865)$ & $29(7-120)$ \\
\hline Negative likelihood ratio $(95 \% \mathrm{Cl})$ & $0.37(0.28-0.49)$ & $0.09(0.01-0.30)$ & $0.42(0.27-0.64)$ & $0.42(0.27-0.65)$ \\
\hline Positive predictive value, $\%(95 \% \mathrm{Cl})$ & $94(84-99)$ & $100(83-100)$ & $94(73-100)$ & $87(60-98)$ \\
\hline Negative predictive value, $\%(95 \% \mathrm{Cl})$ & $94(91-96)$ & $98(95-100)$ & $94(90-97)$ & $87(79-92)$ \\
\hline
\end{tabular}

$\mathrm{Cl}=$ confidence interval.

*Swab obtained within 48 hours before isolate collection.

†Swab obtained between 48 hours and 14 days before isolate collection.

$\neq$ Swab obtained more than 14 days before isolate collection.

$\S$ Test characteristics could not be calculated owing to high specificity values.

Table 3: Test characteristics of screening to predict methicillin resistance in clinical isolates of Staphylococcus aureus, stratified by sterility of site, intensive care unit admission at the time of isolate collection, and screening swab and isolate collection on same admission

\begin{tabular}{|c|c|c|c|c|c|c|}
\hline \multirow[b]{2}{*}{ Characteristic } & \multicolumn{2}{|c|}{ Site of isolate collection } & \multicolumn{2}{|c|}{ Admission } & \multicolumn{2}{|c|}{$\begin{array}{l}\text { Screening swab and isolate } \\
\text { collection }\end{array}$} \\
\hline & Sterile & Nonsterile & ICU & Non-ICU & Same admission & $\begin{array}{l}\text { Different } \\
\text { admission }\end{array}$ \\
\hline True positive, no. & 5 & 45 & 17 & 33 & 43 & 7 \\
\hline True negative, no. & 97 & 331 & 179 & 249 & 365 & 63 \\
\hline False positive, no. & 1 & 2 & 0 & 3 & 1 & 2 \\
\hline False negative, no. & 6 & 23 & 11 & 18 & 20 & 9 \\
\hline $\begin{array}{l}\text { Sensitivity, \% } \\
(95 \% \mathrm{Cl})\end{array}$ & $45(17-77)$ & $66(54-77)$ & $61(41-79)$ & $65(50-78)$ & $68(55-79)$ & $44(31-62)$ \\
\hline $\begin{array}{l}\text { Specificity, \% } \\
(95 \% \mathrm{Cl})\end{array}$ & $99(94-100)$ & $99(98-100)$ & $100(98-100)$ & $99(97-100)$ & $99(98-100)$ & $97(89-100)$ \\
\hline $\begin{array}{l}\text { Positive likelihood } \\
\text { ratio }(95 \% \mathrm{Cl})\end{array}$ & $45(5.7-348)$ & $111(27-443)$ & $-*$ & $54(17-170)$ & $250(35-1782)$ & $14(3.3-62)$ \\
\hline $\begin{array}{l}\text { Negative likelihood } \\
\text { ratio }(95 \% \mathrm{Cl})\end{array}$ & $0.55(0.32-0.95)$ & $0.34(0.24-0.47)$ & $0.39(0.21-0.6)$ & $0.36(0.25-0.52)$ & $0.32(0.22-0.46)$ & $0.58(0.38-0.9)$ \\
\hline $\begin{array}{l}\text { Positive predictive } \\
\text { value, } \%(95 \% \mathrm{Cl})\end{array}$ & $83(36-100)$ & $96(86-100)$ & $100(80-100)$ & $92(77-98)$ & $98(88-100)$ & $78(40-97)$ \\
\hline $\begin{array}{l}\text { Negative predictive } \\
\text { value, } \%(95 \% \mathrm{Cl})\end{array}$ & $94(88-98)$ & $94(90-96)$ & $94(90-97)$ & $93(90-96)$ & 95 (92-97) & $88(78-94)$ \\
\hline
\end{tabular}


results of isolate resistance were available. In these cases, the screening results were available earlier than the isolate results by a mean of 2.1 $( \pm 1.2)$ days.

\section{Interpretation}

In our population, we found that a positive result on screening for MRSA was associated with a positive likelihood ratio of 91 and a positive predictive value of $94 \%$ for subsequent methicillin resistance in an infection with $S$. aureus. High positive likelihood ratios persisted with time and across different admissions. Overall sensitivity of screening for MRSA was poor (63\%); however, sensitivity was excellent (91\%) if the swab was obtained within 48 hours before collection of the clinical isolate. In this scenario, the negative likelihood ratio was 0.09 , yielding a negative predictive value in our population of $98 \%$. The sensitivity declined with increasing time between swab and isolate collection.

Few studies to date have examined the predictive value of screening for MRSA. Most were limited to swabs obtained either on admission ${ }^{23}$ or within 24 hours of a clinical infection, and included all clinical infections. ${ }^{19}$ Strymish and colleagues ${ }^{24}$ studied the utility of prior screening in predicting the results of preoperative screening for MRSA, and found a similar specificity to that found to our study. In our study, the persistence of high specificity is likely due to the duration of MRSA carriage, with median colonization of 6 months, ${ }^{25}$ although the maximum time between swab and isolate collection seen in this study was 569 days. Loss of sensitivity after 48 hours is likely due to new acquisition of MRSA, given that in one study MRSA colonization was associated with a hospital stay of $18( \pm 8)$ days. ${ }^{22}$ In our study's population, most of the screening results were available before the results for susceptibility of clinical isolates, with a mean difference of 2.1 days. This is an important finding for stewardship programs, because it represents valuable time when treatment could be changed to MSSAspecific $\beta$-lactam antibiotics, which have superior cure rates to vancomycin in sensitive strains. ${ }^{6}$ The value of negative results on MRSA screening applies only to regions with low prevalence of MRSA, such as Canada and many European countries. ${ }^{26}$ Further research is needed to clarify the utility of negative results from swabs collected more than 48 hours before infection.

\section{Limitations}

A limitation of this study is that we derived test characteristics from cases of culture-positive infection. By definition, we cannot determine the
Table 4: Multivariate analysis of predictors of concordance between methicillin resistance on screening and Staphylococcus aureus clinical isolate

\begin{tabular}{|c|c|}
\hline Variable* & OR $(95 \% \mathrm{Cl})$ \\
\hline Specimen taken from sterile site & $1.0 \quad(0.4-2.5)$ \\
\hline Antibiotics given between swab and isolate collection & $1.0 \quad(0.4-2.1)$ \\
\hline Age, yrt & $1.0(0.98-1.02)$ \\
\hline ICU v. non-ICU admission & $1.2 \quad(0.5-2.7)$ \\
\hline Sex (male v. female) & $1.3 \quad(0.6-2.7)$ \\
\hline Surgical v. medical unit & $0.9 \quad(0.4-1.8)$ \\
\hline Time between admission and isolate collection, $d \neq$ & $1.0(0.99-1.0)$ \\
\hline Swab and isolate collection on same admission & $2.1 \quad(0.9-4.6)$ \\
\hline Swab obtained within $48 \mathrm{~h}$ of isolate collection & $(1.6-28.2)$ \\
\hline $\begin{array}{l}\mathrm{Cl}=\text { confidence interval, ICU = intensive care unit, } \mathrm{OR}=\text { odds ratio. } \\
\text { *All dichotomous values as yes } \mathrm{v} \text {. no unless stated otherwise. } \\
\text { tOR per } 1 \text { year increase in age. } \\
\text { fOR per day. }\end{array}$ & \\
\hline
\end{tabular}

accuracy of test characteristics of MRSA screening for culture-negative infections. We cannot be certain that all $S$. aureus infections were true infections as opposed to colonizations, but we included only patients who received antistaphylococcal antibiotics around the time of culture collection. Moreover, our results did not significantly differ between sterile and nonsterile sites of isolate collection. Our study was conducted in an area of low MRSA prevalence, but sensitivity, specificity and likelihood ratios are intrinsic test characteristics and are independent of the population prevalence of methicillin resistance. We restricted our screening to higher risk populations, which could potentially exclude some patients colonized by MRSA. However, our criteria for screening were likely sufficiently broad, as indicated by the fact that most screening results were negative. Finally, it is possible that patients may have received prior MRSA decolonization, which we were unable to examine in our study. This may represent a group for which prior MRSA screening is less useful in predicting resistance patterns.

\section{Conclusion}

Our study showed that a history of MRSA colonization on screening had a high specificity for methicillin resistance in subsequent culture-positive clinical infections with $S$. aureus. This result persisted even with swabs and clinical isolates collected more than 14 days apart and on different admissions. In areas of low MRSA prevalence, a negative result on screening for MRSA, where the swab was obtained within 48 hours before isolate collection, was associated with a low likelihood of methicillin resistance. Although originally intended for infection-control 
purposes, our data indicate that MRSA screening offers important information about the likelihood of methicillin resistance in subsequent culturepositive $S$. aureus infections.

\section{References}

1. Wisplinghoff H, Bischoff T, Tallent SM, et al. Nosocomial bloodstream infections in US hospitals: analysis of 24179 cases from a prospective nationwide surveillance study. Clin Infect Dis 2004:39:309-17.

2. Results of the surveillance of methicillin resistant Staphylococcus aureus from 1995 to 2009: a project of the Canadian Nosocomial Infection Surveillance Program. Ottawa (ON): Public Health Agency of Canada; 2011.

3. Klein E, Smith DL, Laxminarayan R. Hospitalizations and deaths caused by methicillin-resistant Staphylococcus aureus, United States 1999-2005. Emerg Infect Dis 2007;13:1840-6.

4. de Kraker ME, Davey PG, Grundmann H; BURDEN study group. Mortality and hospital stay associated with resistant Staphylococcus aureus and Escherichia coli bacteremia: estimating the burden of antibiotic resistance in Europe. PLoS Med 2011; Oct. 8:e1001104

5. Honda H, Krauss M, Coppersmith C, et al. Staphyococcus aureus nasal colonization and subsequent infection in the intensive care unit patients: Does methicillin resistance matter? Infect Control Hosp Epidemiol 2010;31:584-91.

6. Schweizer ML, Furuno JP, Harris AD, et al. Comparative effectiveness of nafcillin or cefazolin versus vancomycin in methicillin-susceptible Staphylococcus aureus bacteremia. BMC Infect Dis 2011;11:297.

7. Kang CI, Kim SH, Park WB, et al. Bloodstream infections caused by antibiotic-resistant gram-negative bacilli: risk factors for mortality and impact of inappropriate initial antimicrobial therapy on outcome. Antimicrob Agents Chemother 2005;49:760-6.

8. Paul M, Kariv G, Goldberg E, et al. Importance of appropriate empirical antibiotic therapy for methicillin-resistant Staphylococcus aureus bacteraemia. J Antimicrob Chemother 2010;65:2658-65.

9. Fridkin SK, Lawton R, Edwards JR, et al. Monitoring antimicrobial use and resistance: comparison with a national benchmark on reducing vancomycin use and vancomycin-resistant enterococci. Emerg Infect Dis 2002;8:702-7.

10. Roghmann MC. Predicting methicillin resistance and the effect of inadequate empiric therapy on survival in patients with Staphylococcus aureus bacteremia. Arch Intern Med 2000;160:1001-4.

11. Clavo-Sánchez AJ, Girón-González JA, López-Prieto D, et al Multivariate analysis of risk factors for infection due to penicillin-resistance and multidrug-resistant Streptococcus pneumoniae: a multicenter study. Clin Infect Dis 1997;24:1052-9.

12. Chiang WC, Chen SY, Chien KL, et al. Predictive model of antimicrobial-resistant gram-negative bacteremia at the ED. Am J Emerg Med 2007;25:597-607.

13. Peña C, Pujol M, Ricart A, et al. Risk factors for faecal carriage of Klebsiella pneumoniae producing extended spectrum betalactamase (ESBL-KP) in intensive care unit. J Hosp Infect 1997; 35:9-16.

14. Christiaens G, Ciccarella Y, Damas P, et al. Prospective survey of digestive tract colonization with Enterobacteriaceae that produce extended-spectrum b-lactamases in intensive care unit J Hosp Infect 2006;62:386-8.

15. Robicsek A, Beaumont JL, Paule SM, et al. Universal surveil- lance for methicillin-resistant Staphylococcus aureus in 3 affiliated hospitals. Ann Intern Med 2008;148:409-18.

16. Jain R, Kralovic SM, Evans ME, et al. Veterans Affairs initiative to prevent methicillin-resisitant Staphylococcus aureus infections. N Engl J Med 2011;364:1419-30.

17. Davis KA, Stewart JJ, Crouch HK, et al. Methicillin-resistant Staphylococcus aureus (MRSA) nares colonization at hospital admission and its effect on subsequent MRSA infection. Clin Infect Dis 2004;39:776-82.

18. Robicsek A, Suseno M, Beaumont J, et al. Prediction of methicillin-resistant Staphylococcus aureus involvement in disease site by concomittant nasal sampling. J Clin Microbiol 2008;46: 588-92.

19. von Eiff C, Becker K, Machka K, et al. Nasal carriage as a source of Staphylococcus aureus bacteremia. N Engl J Med 2001; 344:11-6.

20. Corne $\mathrm{P}$, Marchandin $\mathrm{H}$, Jonquet $\mathrm{O}$, et al. Molecular evidence that nasal carriage of Staphylococcus aureus plays a role in respiratory tract infections in critically ill patients. J Clin Microbiol 2005;43:3491-3

21. Elligsen M, Walker S, Simor A, et al. Prospective audit and feedback of an antimicrobial stewardship in critical care: program implementation, experience, and challenges. Can J Hosp Pharm 2012;65:31-6

22. Fishbain JT, Lee JC, Nguyen HD, et al. Nosocomial transmission of methicillin-resistant Staphylococcus aureus: a blinded study to establish baseline acquisition rates. Infect Control Hosp Epidemiol 2003;24:415-21.

23. Harris AD, Furuno JP, Roghmann MC, et al. Targeted surveillance of methicillin-resistant Staphylococcus aureus and its potential use to guide empiric antibiotic therapy. Antimicrob Agents Chemother 2010;54:3143-8.

24. Strymish J, Branch-Elliman W, Itani K, et al. Clinical history of methicillin-resistant Staphylococcus aureus is a poor predictor of preoperative colonization status and post-operative infections. Infect Control Hosp Epidemiol 2012;33:1113-7.

25. Larsson AK, Gustafsson E, Nilsson AC, et al. Duration of methicillin-resistant Staphylococcus aureus colonization after diagnosis: a four-year experience from southern Sweden. Scand J Infect Dis 2011;43:456-62.

26. Johnson AP. Methicillin-resistant Staphylococcus aureus: the European landscape. J Antimicrob Chemother 2011;66(Suppl 4):iv43-8.

Affiliations: Division of Infectious Diseases (MacFadden, Ricciuto, Daneman), University of Toronto; Division of Infectious Diseases (Elligsen, Daneman) Sunnybrook Health Sciences Centre, Toronto, Ont.; Division of Infectious Diseases (Robicsek), University of Chicago, Chicago, Ill.; Institute for Clinical Evaluative Sciences (Daneman), Toronto, Ont.

Contributors: Derek MacFadden, Daniel Ricciuto and Nick Daneman contributed to the conceptualization and design of the study. Derek MacFadden, Marion Elligsen and Nick Daneman conducted the analysis. All authors contributed to the interpretation of the results. Derek MacFadden drafted the manuscript, which all of the authors revised. All of the authors approved the final version submitted for publication.

Acknowledgement: The authors acknowledge Steven Shumak for inspiring this study with a poignant question on the wards. 